\title{
Mesoscopic Simulations of Unsteady Shear-Thinning Flows
}

\author{
Abdel Monim Artoli and Adélia Sequeira \\ Centre for Mathematics and its Applications (CEMAT) \\ Departamento de Matemática, Instituto Superior Técnico \\ Av. Rovisco Pais, 1049-001 LISBOA \\ Portugal \\ Tel.: +35121 8417073; Fax: +351 218417048 \\ \{artoli, adelia.sequeira\}@math.ist.utl.pt \\ http://www.math.ist.utl.pt/cemat/
}

\begin{abstract}
The capability of the lattice Boltzmann method as an accurate mesoscopic solver for unsteady non-Newtonian flows is shown by investigating pulsatile shear-thinning blood flow in a three-dimensional idealised vessel. The non-Newtonian behaviour of blood flow is modelled by the Carreau-Yasuda viscosity model. Higher velocity and shear stress magnitudes, relative to Newtonian cases, are observed for the shear-thinning simulations in response to changes in the shear-rate dependent Womersley parameter. We also investigate the flexibility of the method through the shear-thinning behaviour of the lattice Boltzmann relaxation parameter at different Deborah numbers.
\end{abstract}

\section{Introduction}

The role of hemodynamics on the development of cardiovascular diseases and the high rate of failure in vascular implantation of artificial devices is still under research focus, as these diseases are a major cause of mortality in the world [1]. Since long, analytical, numerical and experimental investigations of steady and pulsatile blood flow of Newtonian and non-Newtonian models have been carried out (see e.g. [2] for a review). It is now well established that localised low and oscillatory shear stress and high shearrate gradients promote, among other factors, most of the diseases of the cardiovascular system.

With recent advances in mathematical modelling and computer technology, research in this field is now very active, targeting a better understanding of the complex nature of the fluid and the transporting vessels. Blood is a heterogeneous suspension of small deformable red blood cells, white blood cells, platelets and other matter in plasma. Red blood cells characterise the main rheological behaviour of the fluid. These cells aggregate and deform in response to the applied shear rate, change the fluid viscosity and relax back within about $100 \mathrm{msec}$. The non-Newtonian behaviour of many fluids, including blood, may be well described by the shear-thinning Carreau-Yasuda viscosity model

$$
\eta=\eta_{\infty}+\left(\eta_{0}-\eta_{\infty}\right)\left(1+(\lambda \dot{\gamma})^{a}\right)^{b}
$$


where $\eta_{0}$ and $\eta_{\infty}$ are the asymptotic low and high shear-rate viscosities, respectively, $\lambda$ is a characteristic viscoelastic time of the fluid, $\dot{\gamma}$ is the shear rate and the parameters $a$ and $b$ are determined from experimental data, with $b<0$ for the shear thinning fluids. For blood, $\eta_{0}=0.022 \mathrm{~Pa} \mathrm{~S}, \eta_{\infty}=0.0022 \mathrm{~Pa} \mathrm{~S}, \lambda=0.11 \mathrm{~S}, a=0.644$ and $b=-0.944099$ (see e.g. [3]). The fluid behaves as Newtonian for small $\dot{\gamma}$ or negligible values of $\lambda$ and also when $a=0$ or $b=0$.

In addition to hemodynamics, many industrial activities involve shear-thinning fluid processing and numerical simulations are found viable. In most numerical studies, accurate and robust conventional Navier-Stokes solvers are required to simulate flow of shear-thinning fluids [4]. However, these solvers yield three major difficulties: the nontrivial and time consuming grid generation, the necessity to solve the Poisson equation for the pressure field with additional corrective terms, and the approximations involved in computing the shear rate from computed velocity field. All these shortcomings are eliminated with the newly established lattice Boltzmann method (LBM). Firstly, LBM works with a Cartesian grid, making it highly suitable for high performance computing. Secondly, the pressure is directly computed from the linear equation of state without a need to solve the Poisson equation. Last and more important in computational rheology is that the shear rate is directly obtained from the non-equilibrium part of the distribution functions, independent of the velocity field. This adds a great flexibility to the LBM in modelling and simulation of most non-Newtonian fluids, as it is easy to establish constitutive equations with the use of simple equations. This will be explained in the next section.

In a previous article [5], it has been demonstrated that LBM is an accurate and robust hemodynamic numerical solver for unsteady Newtonian flows in realistic geometries. Localised shear stress and velocity field have well compared with values in the available literature. Following arguments that the non-Newtonian influence should not be ignored, the main objective of this study is to explore in general the capability of the lattice Boltzmann method in simulating shear-thinning flows. A number of lattice Boltzmann simulations of shear-thinning flows using the Casson viscosity model have been reported in the literature [6, 7]. Results on LBM simulations of 3D Carreau-Yasuda flows are lacking. Due to the limitations of Casson model for blood in particular, the Carreau-Yasuda model is adopted in this study.

In the rest of this paper, we will briefly describe the adaptation of LBM to nonNewtonian shear-thinning fluids and present our simulation results for a benchmark test of three-dimensional pulsatile blood flow in a straight tube. Gijsen et al.[3] have compared shear thinning results with analytical Newtonian solutions, finite elements simulations and experimental results and demonstrated the deviation of shear-thinning flow from Newtonian solutions. Taking this procedure into account, here we compare the obtained results with analytical and simulated results. Comparison with other CFD methods is a subject of ongoing research.

\section{The Lattice Boltzmann Method for Shear-Thinning Flows}

The lattice Boltzmann method is a special finite difference discretisation of the simplified Boltzmann equation [8, 9, 10] which describes transport phenomena at the 
mesoscale level. The dynamics of the fluid is modelled by the transport of simple fictitious particles on the nodes of a Cartesian grid. Simulations with this method involve two simple steps; streaming to the neighbouring nodes and colliding with local node populations represented by the probability $f_{i}$ of a particle moving with a velocity $\mathbf{e}_{i}$ per unit time step $\delta t$. Populations are relaxed towards their equilibrium states during a collision process. The equilibrium distribution function

$$
f_{i}^{(e q)}=w_{i} \rho\left(1+\frac{3}{v^{2}} \mathbf{e}_{i} \cdot \mathbf{u}+\frac{9}{2 v^{4}}\left(\mathbf{e}_{i} \cdot \mathbf{u}\right)^{2}-\frac{3}{2 v^{2}} \mathbf{u} \cdot \mathbf{u}\right)
$$

is a low Mach number approximation to the Maxwellian distribution. Here, $w_{i}$ is a weighting factor, $v=\delta x / \delta t$ is the lattice speed, and $\delta x$ is the lattice spacing.

The lattice Boltzmann equation

$$
f_{i}\left(\mathbf{x}+\mathbf{e}_{i} \delta t, \mathbf{e}_{i}, t+\delta t\right)-f_{i}\left(\mathbf{x}, \mathbf{e}_{i}, t\right)=-\frac{1}{\tau}\left[f_{i}\left(\mathbf{x}, \mathbf{e}_{i}, t\right)-f_{i}^{(0)}\left(\mathbf{x}, \mathbf{e}_{i}, t\right)\right]
$$

can be obtained by discretizing the evolution equation of the distribution functions in the velocity space using a finite set of velocities $\mathbf{e}_{i}$. In this equation, $\tau$ is the dimensionless relaxation time. By Taylor expansion of the lattice Boltzmann equation up to $O\left(\delta t^{2}\right)$ and application of the multiscale Chapman-Enskog technique [10], the NavierStokes equations and the momentum flux tensor up to second order in the Knudsen number are obtained. The hydrodynamic density, $\rho$, and the macroscopic velocity, $\mathbf{u}$, are determined in terms of the particle distribution functions from the laws of conservation of mass and momentum: $\rho=\sum_{i} f_{i}=\sum_{i} f_{i}^{(e q)}$ and $\rho \mathbf{u}=\sum_{i} \mathbf{e}_{i} f_{i}=\sum_{i} \mathbf{e}_{i} f_{i}^{(e q)}$. The pressure is given by $p=\rho c_{s}^{2}$ and the kinematic viscosity is $v=c_{s}^{2} \delta t\left(\tau-\frac{1}{2}\right)$, where $c_{s}$ is the lattice speed of sound. A number of lattice Boltzmann models have been introduced, being characterised by the choice distribution functions, the number of moving particles and the lattice speed of sound. Furthermore, by expanding $f_{i}$ about its equilibrium distribution $f_{i}^{0}$

$$
f_{i}=f_{i}^{0}+\varepsilon f_{i}^{(1)}+\varepsilon^{2} f_{i}^{(2)}+\ldots
$$

where $\varepsilon$ is of the order of the Knudsen number and in the limit of small $\varepsilon$, the momentum flux tensor is obtained by [10]

$$
\Pi_{\alpha \beta}^{(1)}=\sum_{i} f_{i}^{(1)} \mathbf{e}_{i \alpha} e_{i \beta}=-2 \rho \delta_{t} \tau_{c} C\left(S_{\alpha \beta}\right),
$$

where $C$ is a lattice-dependent constant (for the three-dimensional model with 19 particles (D3Q19), $C=1 / 3$ ). The momentum flux is therefore directly computed from the non-equilibrium part of the distribution functions and therefore, the strain rate tensor is

$$
S_{\alpha \beta}=-\frac{1}{2 C \delta_{t} \tau_{c} \rho} \sum_{i} f_{i}^{(1)} \mathbf{e}_{i \alpha} e_{i \beta}
$$

and the stress tensor from

$$
\sigma_{\alpha \beta}=-\rho c_{s}^{2} \delta_{\alpha \beta}-\left(1-\frac{1}{2 \tau_{c}}\right) \sum_{i=0} f_{i}^{(1)} e_{i \alpha} e_{i \beta} .
$$


In constitutive equations of shear-thinning generalised Newtonian fluids the viscosity depends on the magnitude or the second invariant of the strain rate tensor which can be computed from the double inner product of $S_{\alpha \beta}$ by itself

$$
|S| \equiv \dot{\gamma}=\sqrt{2 S_{\alpha \beta}: S_{\alpha \beta}}
$$

More specifically, for the $D 3 Q 19$ model, after making use of the symmetry of the strain rate tensor, this is locally computed during collision from the simple relation

$$
\dot{\gamma}=\dot{\gamma}_{c} \sqrt{0.5\left(S_{x x}^{2}+S_{y y}^{2}+S_{z z}^{2}\right)+\left(S_{x y}^{2}+S_{x z}^{2}+S_{y z}^{2}\right)}
$$

where $\dot{\gamma}_{c}=\frac{3}{2 \rho \tau_{c}}$ could be used as a characteristic shear rate. In this study we propose that $\tau_{c}=1$ to benefit from the simplicity and the accuracy of the scheme at $\tau=1$ since the relaxation towards equilibrium in this case (for Newtonian fluids) is direct. This will allow us to avoid rescaling dimensionless numbers when Newtonian solutions are simulated for comparison. In this article the Carreau-Yasuda model is used (see Eq. 1) to model non-Newtonian blood viscosity. Other non-Newtonian characteristics of blood may be ignored [3]. In LBM simulations, we can implement the CarreauYasuda model in terms of the dimensionless relaxation time. Making use of the fact that $\eta=\rho v=\rho(2 \tau-1) / 6$, the Carreau-Yasuda model in its dimensionless form is

$$
\tau=\tau_{\infty}+\left(\tau_{0}-\tau_{\infty}\right)\left(1+(\lambda \dot{\gamma})^{a}\right)^{b}
$$

where $\tau_{0}$ and $\tau_{\infty}$ correspond to $\eta_{0}$ and $\eta_{\infty}$, respectively. The stability of the method is controlled by the difference $\left(\tau_{0}-\tau_{\infty}\right)$ which is normally large for shear-thinning fluids. This slightly enhances the stability of the LBM scheme as one more free parameter is introduced. However, $\tau_{\infty}$ shall be in the working stability region if $\tau_{0}$ is small. Another way to avoid instability is to tune the material relaxation time (in lattice units) by gridrefinement (or coarsening) in time.

The variety of numerous dimensionless numbers defined for the shear-thinning fluids implies great restrictions on the choice of working free parameters and has motivated research towards specialised LBM schemes for viscoelasticity [11]. However, for shearthinning fluids, the standard BGK [8] algorithm allows us to introduce two additional parameters, one for the high shear-rate viscosity and the other for the difference between high-shear rate and low-shear rate viscosities. This adds a great flexibility to the lattice BGK method and it would not be necessary to go beyond the BGK for shear-thinning fluids. This might not be the case for other viscosity models.

\section{Simulations}

We have conducted a number of benchmark simulations for pulsatile shear-thinning blood flow in a three-dimensional rigid straight tube with circular cross-section of constant radius. The main objective of this simulation is to investigate the influence of non-Newtonian behaviour on the velocity field and the shear stress. The shearthinning behaviour is captured by the Carreau-Yasuda model explained in the previous section. The diameter $D$ of the tube is represented by 20 lattice points and the 


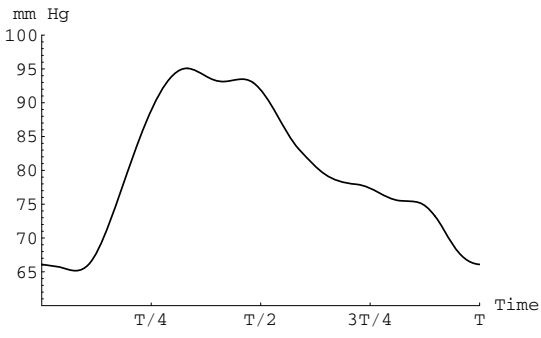

(a)

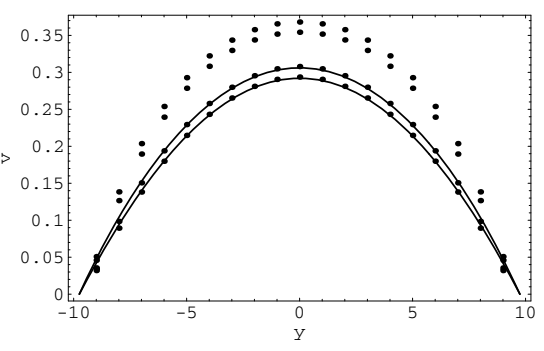

(b)

Fig. 1. (a) Pressure pulse and (b) LBM unsteady shear-thinning velocity profiles at $0.2 \mathrm{~T}$ (upper dots, lower values) and $0.3 \mathrm{~T}$ (upper dots, higher values) compared to analytical Newtonian solutions (lines). Shown are also Newtonian simulations (lower dots attached to the lines).

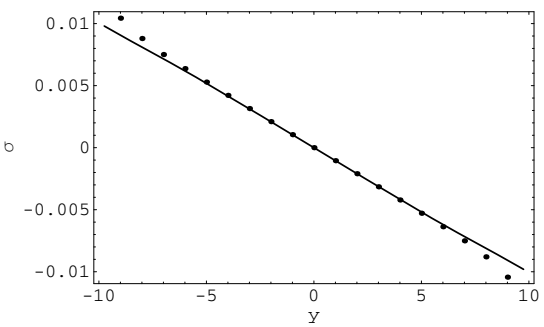

Fig. 2. LBM shear stress results (dots) for pulsatile shear-thinning blood flow at the mid-diastole compared to the analytical Newtonian solution (line)

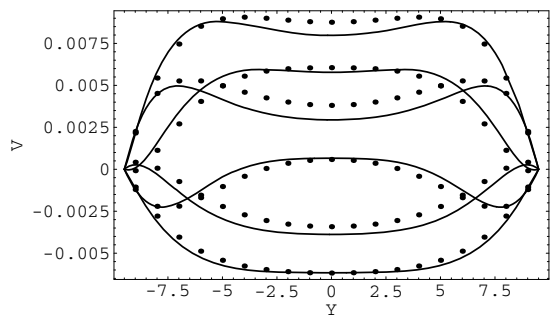

Fig. 3. Simulated (dots) harmonic velocity components compared to the analytical Newtonian solutions (lines)

period $T$ of the pressure pulse by 100 time steps. The dimensionless numbers are $D e=\frac{\lambda}{T}=0.1164, C r=0.2, \operatorname{Re}=U D / v=42$ and $\alpha=D / 2 \sqrt{\frac{2 \pi}{T v}}=6.2$ at their maximum values, where $\lambda$ is the material parameter, $D e$ is the Deborah number, $C r$ is the Carreau number, $R e$ is the Reynolds number and $\alpha$ is the Womersley number. The boundary conditions are applied as follows. The bounce-back boundary condition is applied on the walls. A pressure gradient computed from a pulse (see Fig. 1 (a)) measured at the entrance of the human carotid artery is Fourier transformed into a steady and 16 harmonic terms and applied at the entrance of the tube as an inlet condition [5]. At the outlet, a constant pressure is implemented. The velocity and unknown distributions at the 


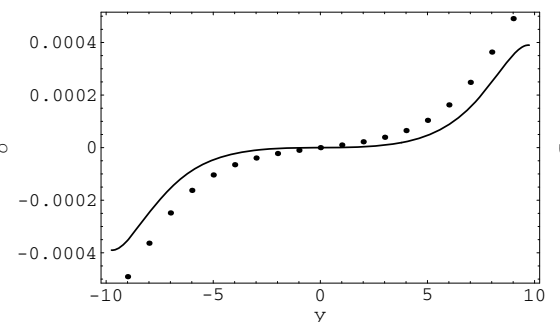

(a)

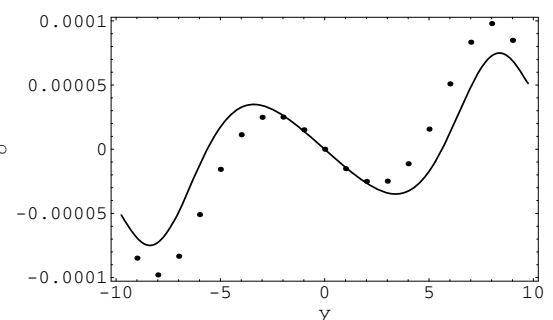

(b)

Fig. 4. Deviation from Newtonian profile for simulated (dots) harmonic shear-stress at (a) the begining of systole and (b) at the mid-diastole

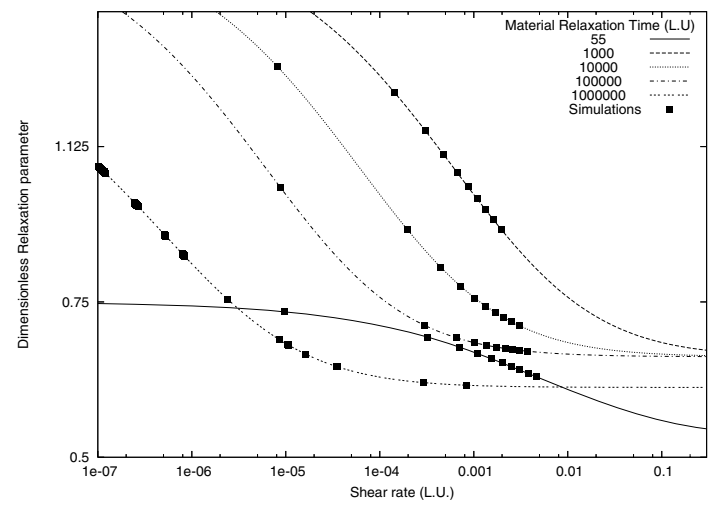

Fig. 5. Shear-thinning behavior of the LBM relaxation time for different material relaxation times

boundaries are computed from the implemented pressure boundary conditions. Figure 1 (b) shows simulation results for the shear-thinning (upper dots) flow case compared to the analytical Newtonians (lines). Newtonian simulation results are also shown as dots on the lines demonstrating an excellent agreement with the theory. The unsteady non-Newtonian profile is almost parabolic with slightly varying amplitudes throughout the cardiac cycle. The difference between Newtonian and non-Newtonian results varies in time and across the tube with a maximum of $20 \%$ at the centerline. We have observed significant deviations from the non-Newtonian in the shear stress close to the walls through the whole cardiac cycle, with a maximum difference at the mid-diastole (see Fig. 2), in qualitative agreement with shear-thinning results compared to Newtonian solutions and experiments in Ref. [3]. The comparison with Newtonian cases is not meant for direct validation but by transitivity as the study by Gijsen et al. [3] have compared numerical and experimental values for velocity and shear stress deviation of Carreau-Yasuda fluids with Newtonian solutions.

The pressure pulse can be splitted into a steady mean component and oscillatory components. For better understanding of the problem, we have performed a number of steady and oscillatory simulations. The steady component produced a simple parabolic profile for the Newtonian case and a semi-parabolic profile with higher amplitude for 
the shear-thinning case (data not shown). However, the flow derived by oscillatory components shows significant deviation from the Newtonian behavior for both velocity (Fig.3) and shear stress (Figs. 4 (a) and (b)). These deviations are attributed to changes in $\alpha$ in response to changes in the computed shear rate. For small values of $\alpha$, the difference is more significant in the center for velocity (see Fig. 3) and close to the walls for the shear stress (see Figs. 4 ). For larger $\alpha$ (as in the aorta) the situation is reversed; more flattened profiles in the center with differences close to the walls for the velocity and shear stress (data not shown).

In general, for this specific benchmark, the non-Newtonian shear-thinning flow gives higher velocities and higher shear stress values than when the fluid is considered Newtonian. The oscillatory profile of the shear stress, controlled by the Womersley number behaves almost similarly, with peaks more distant from the walls than for the Newtonian cases, as is clearly shown in Fig. 4.

To investigate the flexibility of LBM in simulating flow of a shear-thinning fluid, we have explored possible stable solutions at different shear rates with different values of $\lambda_{L}$ (different $D e$ numbers) and recorded the dimensionless LBM relaxation time. Figure 5 shows the shear-thinning behavior of the LBM relaxation time. From this figure we observe that the dimensionless relaxation time is flexible enough in simulations of shear thinning flows as the shear rate changes six orders of magnitude. For a fluid like blood, at most three orders of magnitude changes in the shear rate have been reported and therefore, the scheme is quite suitable as a shear-thinning hemodynamic solver. For other polymeric fluids we can argue similar conclusions.

\section{Conclusions}

Unsteady non-Newtonian shear-thinning blood flow in a straight rigid tube is investigated using the Carreau-Yasuda model and simulated with the lattice Boltzmann method. A characteristic relaxation time has been added to the standard BGK scheme to avoid implicit dependency of the strain rate and the relaxation parameter. The capabilities of the method as an efficient and accurate numerical solver for this type of problems have been successfully demonstrated and benefits over the commonly used Navier-Stokes solvers have been highlighted. The most relevant advantage in using this numerical solver lies in the fact that the shear rate is directly computed from the non-equilibrium part of the distribution functions without a need for computing the velocity gradients. This enables more sophisticated shear-rate dependent fluid viscosities to be modeled accurately and efficiently. Comparison with other numerical methods is underway. Future work would involve simulating shear-thinning fluid flows in realistic geometries from hemodynamics and industrial applications.

\section{Acknowledgments}

This work has been partially supported by the grant SFRH/BPD/20823/2004 of Fundação para a Ciência e a Tecnologia (A. Artoli), by the Center for Mathematics and its Applications - CEMAT through FCTs funding program and the projects HPRN-CT2002-00270 and POCTI/MAT/41898/ 2001. 


\section{References}

1. The World Health Report 2005, WHO publications (2005).

2. Wooton, D.M., Ku, D.N.: Fluid mechanics of vascular systems, diseases, and thrombosis. Annual Review of Biomedical Engineering 1 (1999) 299-329.

3. Gijsen, F.J.H., de Vosse, F.N., Janssen F.N.: The influence of the non-Newtonian properties of blood on the flow in large arteries: steady flow in a carotid bifurcation model. Journal of Biomechanics 32 (1999) 601-608.

4. Arada, N., Pires, M., Sequeira, A.: Numerical simulations of shear-thinning Oldroyd-B fluids in curved pipes. IASME Transactions 62 (2005) 948-959.

5. Artoli, A.M., Hoekstra A.G., Sloot P.M.A.: Simulation of a Systolic Cycle in a Realistic Artery with the Lattice Boltzmann BGK Method, International Journal of Modern Physics B, 17 (2003) 95-98.

6. Ouared, R.,Chopard B.; Lattice Boltzmann Simulations of Blood Flow: Non-Newtonian Rheology and Clotting Processes. Journal of Statistical Physics 121 1-2 (2005) 209- 221.

7. Aharonov, E., Rothman, D.H.:Non-newtonian flow (through porous media): a lattice boltzmann method. Geophys. Research Letters 20 (1993) 679-682.

8. Sauro Succi: The Lattice Boltzmann Equation for Fluid Dynamics and Beyond. Oxford University Press (2001).

9. Benzi R., Succi S., Vergassola M.: The Lattice Boltzmann Equation - Theory and Applications. Physics Reports 222 (1992) 145-197.

10. Chopard B. and Droz M.: Cellular Automata Modeling of Physical Systems. Cambridge University Press (1998).

11. Giraud, L., d'Humires, D., Lallemand, P.: A lattice Boltzmann model for visco-elasticity. International Journal of Modern Physics C 8 (1997) 805-815. 